ORIGINAL ARTICLE

\title{
COMPARISON OF MCKENZIE APPROACH VERSUS LUMBAR STABILIZATION EXERCISES IN THE TREATMENT OF CHRONIC LOW BACK PAIN
}

Faria Aqil ${ }^{1}$, Muhammad Affan Iqbal ${ }^{1}$, Suhail Karim ${ }^{1}$, Muhammad Umar Iqbal ${ }^{2}$, Muhammad Junaid Akram², Zona Mehreen $^{3}$, Raeed Mufti ${ }^{1}$

\author{
Submitted: October 15, 2020 \\ Accepted: April 25, 2021 \\ Published: June 30, 2021
}

Authors' Affiliation
${ }^{1}$ Riphah International
University, Islamabad
${ }^{2}$ Shifa Tameer-e-Millat
University, Islamabad
${ }^{3}$ Isra Institute of Rehabilitation
Sciences, Isra University
Islamabad
Corresponding Author
Raeed Mufti
${ }^{1}$ Riphah
University, Islamabad
E-mail:
raeed.mufti@gmail.com

\section{ABSTRACT \\ Introduction: Lumbar stabilization and McKenzie exercises are becoming a popular trend for managing chronic low back pain though it is currently not known whether lumbar stabilization exercises produce better results in improving functional status compared to McKenzie approach. The purpose of this study was to determine the effectiveness of the McKenzie approach versus lumbar stabilization in the treatment of chronic low back pain.}

Material \& Methods: This was a randomized controlled trial. Lottery method was used to randomly divide individuals who fulfilled inclusion criteria into 2 groups. Assessments of the patients were done in OPD. Baseline assessment was done on day 1 and post intervention assessment was done after 2 weeks. A total of 8 treatment sessions spread across 2 weeks were given (4 days per week). Data were assessed at baseline level and post intervention. Data was entered and analysed by SPSS version 21 .

Results: A total of 28 females and 02 males (mean age of $50.88 \pm 12.29$ ) participated in the study. After 02 weeks of intervention, both treatment groups showed improvement in decreasing pain and improving functional status. The lumber stabilization group showed significant gains on Numeric Pain Rating Scale and $(p=0.001)$ and Modified Oswestry Disability Index $(p=0.001)$ compared to the Mckenzie group.

Conclusion: Patients in both lumber stabilisation and McKenzie groups showed significant improvement, however, the patients in lumbar stabilization group were superior than the patients in McKenzie group on the selected outcomes.

Key Words: Chronic Low Back Pain, Lumbar Stabilization Exercises, Transversus Abdominis, Oswestry Disability Index

The authors declare no conflict of interest and agree to be accountable for all aspects of the work in ensuring that questions related to the accuracy or integrity of any part of the work are appropriately investigated and resolved. All authors contributed substantially to the planning of research, question designing, data collection, data analysis and write-up of the article.

This article may be cited as: Aqil F, Iqbal MA, Karim S, Iqbal MU, Akram MJ, Mehreen Z, Mufti R. Comparison of Mckenzie approach versus Lumbar Stabilization exercises in the treatment of chronic low back pain. Rehman J Health Sci. 2021;3(1). 24-31

\section{INTRODUCTION}

Low back pain is the most common symptom experienced by all age group people. ${ }^{1}$ The prevalence of low back pain has been reported 80 to $85 \%$ and the incidence of lumbar radiculopathy related to low back pain has been reported 3 to $5 \%$. The unnecessary load on the vertebral column is the most common risk factor of radiculopathy. Most of the people with low back pain have neurological symptoms in the lower limb. Latest studies have emphasized that low back pain is one of the complex disorders that can be influenced by many other factors included cognitive, psychological, physical and lifestyle factors. ${ }^{2}$ These factors have been reported to significantly effect outcomes of rehabilitation program in patients with low back pain.

The number of physician visits by the patients for the treatment of low back pain has increased markedly over the years. In the year 2012, more than 52.3 million patients visited physicians with a complaint of low back pain compared to 44.6 million for the year 2004 in the United States. Spine is the mainstay for a body, allowing standing, bending and twisting while protecting the spinal cord from injury. Body weight is predominantly born by the lumbar spine in the lower back. Degenerative changes are more common in people with the age more than 50 and are even far more prevalent in individuals older than 65 year. Females are more affected by this condition in comparison to males by a ratio of $3: 1$. Back pain may be acute or chronic. Low back pain can be caused by a variety of structures including the spinal muscles, nerves, bones, discs or tendons in the lumbar spine.

Therapeutic exercise for individuals with low back pain has evolved over time. Recently, there has been a focus on exercises that aim to maintain stability in the lumbar spine. ${ }^{3}$ Lumbar stabilization, core stabilization, or segmental stabilization is an active form of exercise used in physical therapy. It is designed to strengthen muscles to support the spine and help prevent lower back pain. Lumbar stabilization is a multi-component program and involves training, strength, flexibility and endurance. It 
is generally used during all phases of a back-pain episode and may be prescribed after a thorough evaluation of the patient's specific condition. Through a regimen of exercises and with the help of an experienced physical therapist, the patient is trained to find and maintain her/his "neutral spine" position. The back muscles are then exercised to teach the spine how to stay in this position. Several groups of muscles are targeted, particularly the Transversus Abdominis (TrA), Lumbar Multifidus, Lumbar Para Spinal, abdominal, diaphragmatic and pelvic musculature.

Richardson et al. presented the first exercise program for the activation and strengthening of the $\operatorname{TrA}$ and multifidus muscles. The program assumed that the power and endurance of TrA and lumbar Multifidus muscles affect the stability of the lumbar spine as a whole and as a result, reduce pain and increase functional outcomes. They emphasized on the co-activation of the TrA and multifidus muscles by isometric contractions and followed by training of these muscles by level progression. ${ }^{3}$

For the activation of TrA and lumbar multifidus muscles patients are instructed to lie supine in hook-lying position while maintaining a neutral spine. The patient is then asked to press the belly button in while exhaling. As the patient isometrically contracts the abdominal muscle, the TrA and lumbar multifidus co-activates. A number of techniques are used for this counter activation of muscles. If a patient has difficulty in the activation of $\operatorname{TrA}$, a pressure biofeedback unit can be used for learning as it gives visual feedback. A small inflatable cuff is placed under the lumbar spine with a pressure gauge in the patient's hand. The cuff is then inflated to $40 \mathrm{mmHg}$ and the patient is asked to draw the belly button while exhaling. When the patient exhales there should be 10 $\mathrm{mmHg}$ raise in the reading. A patient should be able to perform at least 10 isometric holds for 10 seconds each without fatiguing. Once a patient becomes comfortable with this technique, the exercises can be advanced for individual muscle training. ${ }^{4}$

A study conducted in 2010 on segmental stabilization and muscular strengthening suggested that both techniques had lessened pain and reduced disability in patients with chronic low back pain. It has been reported that segmental stabilization has better outcomes compared to superficial strengthening programs. Superficial strengthening program does not improve TrA activation capacity which might be one of the reasons for having inferior outcomes than segmental stabilization. ${ }^{5}$ A study conducted on the effect of lumbar stabilization exercise training on functional ability and quality of life in patients with chronic low back pain suggested that the program of lumbar stabilization is effective in improving quality of life and functional outcome in patients with chronic low back pain. ${ }^{6}$

McKenzie method is one of the most effective programs used for the treatment of patients with nonspecific back pain. This treatment method was developed by Robin McKenzie. There are three stages involved in this program; evaluation, treatment and prophylaxis stages that follow the given bases through inclusive of different categories such as classification of disorders related to spine and extremities, peripheralization, derangement, and dysfunction or postural syndromes. A series of progressive positions are covered by McKenzie's exercises for back pain. Only progress is further preceded as your pain diminishes through many stages one can. Pillows and standing extensions occur through prone lying while resting on elbows, prone lying and prone push-ups. Interpretation of the unique categorization and progressive extension is used in numerous variations of the McKenzie method which improve or confiscate stages accordingly. ${ }^{6}$

The McKenzie method uses an approach that involves postural awareness and repetitive movements with the underlying idea that a converse force can decrease pain and return function. For management of back pain in the short term ( $<3$ months) it is an effective method compared with other therapies suggested by clinical evidences.

Patients with sub-acute or chronic low back pain treated with the intensive dynamic strengthening training and McKenzie method seems to be similarly effective. The patients are classified into four groups according to the mechanical and symptomatic response to repeated movements and sustained positions. Each syndrome demands a different management approach. That is one the reasons that the dilemma which treatment technique is more effective remain unresolved. This study was design to compare lumber stabilisation and McKenzie techniques for the treatment of chronic low back pain.

\section{MATERIAL AND METHODS}

The randomized controlled trial (RCT) was conducted in the rehabilitation department of PAC Kamra, Pakistan. It was completed in 8 months, (i.e. August 2018 till April 2019). A purposive sampling technique was used to collect the sample and randomization was done by using lottery method. The sample size of 30 patients was calculated by OpenEpi tool. Divided into 2 groups; 15 patients in each group. The following study i.e. "Comparison of McKenzie approach versus lumbar stabilization in the treatment of chronic low back pain" was taken as a reference for sample size calculation. ${ }^{8}$ Patients with age ranging from 30 to 60 years with chronic low back pain, radiating pain to the legs and limited SLR were included in the study. Whereas patients with inflammatory condition, postoperative back ache or any soft tissue injuries such as fracture and radiculopathy below the knee were excluded from the study.

Modified Oswestry Disability Index (ODI), Numeric Pain Rating Scale (NPRS) and range of motion (ROM) outcome measures were used to collect data from the patients. Modified ODI is a self-rating outcome tool for low back pain disability. The test is considered as 'Gold Standard' for low back functional outcome. , $^{9,3}$

NPRS is the most ordinary one-dimensional pain measurement scale. The NPRS is the numeric version of Visual Analog Scale (VAS). A horizontal line represents whole number $0-10$, where 0 is represents no pain and 10 represents maximum pain. Individuals have to select a number in-between which reflects the best intensity to their pain. ${ }^{7} \mathrm{ROM}$ is a development capability of a joint to move on its conceivable accessible range. For lumbar spine dynamic movements were performed in standing 
position of a patient. A universal goniometer was utilized to quantify the measure of range of flexion and extension. Measurements were taken just before the start of first session and afterword the last treatment session. ${ }^{8}$ Baseline assessment was done on day 1 and postintervention assessment was done after every 2 weeks. For 2 weeks 8 treatment sessions were given, 4 days per week.

Conventional treatment consisted of TENS (10-15 minutes) and Hot packs (10 minutes). Whereas the Lumbar Stabilization exercises included TrA activation (Figure 1) which was achieved by utilizing drawing manoeuvres. It was started through a snare lying position in the impartial spine and endeavours to keep it up while lumbar stabilization exercises in contracting the stomach muscles. Patients were told to draw the 'belly button' in toward the spine while breathing out. Pressure Biofeedback Unit (PBFU) was utilized to check for the initiation of TrA. Due to non-accessibility to PBFU and high rates for import to Pakistan, we utilized aneroid sphygmomanometer for this purpose. The BP sleeve was put under the lumbar spine and inflate to $40 \mathrm{~mm} \mathrm{Hg}$, the pressure measure was given to the patient and the patient was approached to press the 'belly button' while breathing out. Correct activation was perceived by increase in $10 \mathrm{mmHg}$, whereas greater increase was considered due to pelvic tilt and rectus abdominal activation.

\section{PROGRESSION LEVEL TrA}

Level 1: Patient slouched in recumbent (supine) position with hips and knees flexed and feet on the floor expecting an impartial spine position and endeavoured to keep up it while contracting the stomach muscles. This position was the beginning position for every one of the 5 dimensions of TrA preparation (Figure 2).

Level 2: Starting from the above position, the patient was asked to flex one hip to 90 degrees with knee flex and TrA contracts, return the leg to the starting position and repeat with the opposite leg (Figure 3).

Level 3. While maintaining the position hip flexed 90 degree and knee flex with $\operatorname{TrA}$ contract, bring other leg to same position while maintaining contraction. Then lower one leg following other (Figure 4).

Level 4: Maintaining the position of level 3, the patent was asked to extend one knee without any support and return to last position. Repeat with another leg (Figure 5). Level 5: Without touching surface for support patient was asked to flex both leg and then extend them (Figure $6)$.

The McKenzie method includes few generic exercises and some specific exercises as a treatment protocol and specialized assessment techniques. The exercise protocol was comprising of prone lying, prone press ups, extension in lying, extension in standing, flexion in lying, seated lumbar flexion and standing lumbar flexion. ${ }^{11}$

To check the normality of data Shapiro-Wilk test was applied and $\mathrm{p}$ - value at baseline assessment for NPRS was $p=0.001$, ODI was $p=0.008$, ROM flexion was $\mathrm{p}=0.047$, ROM extension was $\mathrm{p}=0.003$, ROM right side bending was $p=0.048$ and ROM left side bending was $\mathrm{p}=0.008$ which shows data was not normally distributed.
Thus, Mann-Whitney U test (non-parametric) was applied. For various time interim; pre-intervention and post-intervention within the groups Wilcoxon test was applied.

RESULTS

A total of 38 patients were accessed in OPD for eligibility; according to inclusive criteria 30 were included which were divided into two groups, 15 patients in the McKenzie exercise group and 15 in lumbar stabilization exercises group (Figure 7). Four patients were dropped out due to absence of follow-up; 1 from McKenzie group and 3 from lumber stabilisation group. The McKenzie group means age was $52.42 \pm 12.29$ years and lumber stabilisation group mean age was $49 \pm 12.38$ years. Localized nature of pain reported by $18(70.4 \%)$ and transmitting pain $7(29.6 \%)$ among all patients. Nature of pain in the lumber stabilisation group was confined and emanating pain in $8(61.5 \%)$ and $4(38.5 \%)$ patients, respectively.

The comparison at baseline level between LS group and McKenzie groups show non-significant results. At preinterventional level the score of NPRS has median $(\mathrm{IQ}) ; 6(1)$ with non-significant $\mathrm{p}$ value $=0.39$. Variable ODI at pre-interventional level score median (IQ); 49(20.5) having $\mathrm{p}$ value 0.696. Flexion at preintervention level has median (IQ) ;40(2) with p value $=0.568$. At pre-intervention level extension has a median (IQ);15(3), right side rotation 15(5.25) and left side rotation $15(5.5)$ having $\mathrm{p}$ values $0.89,0.655,0.734$, respectively. All these variables showed no differences. Individually both groups showed significant difference; in lumber stabilisation group the NPRS had median IQ at pre-intervention 6.5(1) and post intervention 3(1) with $\mathrm{p}$ value $\mathrm{p}=0.000$, flexions had median IQ at preintervention 42(13) and post intervention 53(7.25) having $\mathrm{p}=0.000$. The median IQ of extension at baseline was 14.5(6) and post intervention was 20(3), right side bending at baseline was 15(7.5) and post intervention was 19(6.5), left side bending at base line 15(6.5) and at post intervention 19(5.5) with $\mathrm{p}=0.000$. The ODI scores $\mathrm{p}=0.000$ having median IQ at base line $49(20.75)$ and post intervention 25(8.75).

In McKenzie group NPRS have median IQ at preintervention was 6(1) and post intervention was 2(2) with $\mathrm{p}=0.000$. Flexion median IQ at pre-intervention was 36.5(4.5) and post intervention 56(5) with $\mathrm{p}=0.000$. The median IQ of extension at baseline was 17(6.75) and post intervention was 25(3), right side bending at baseline was 15(4.5) and post intervention was 20(5.5), left side bending at baseline was 15.5(6.25) and at post intervention was 21(3.5) with $\mathrm{p}=0.000$. The ODI scores with $\mathrm{p}=0.000$ having median IQ at baseline was $49(20.2)$ and post intervention was $28(9.25)$. The two groups show significant improvement on the selected outcomes exclusively, however, lumber stabilisation group indicated superior outcomes than McKenzie exercises.

\section{DISCUSSION}

This study shows that lumbar stabilization exercises are more effective in treating patients with chronic low back pain. Miller et al. conducted RCT on the effects of McKenzie approach and spine stabilization program for 
chronic low back pain and found that the stabilization group illustrated a significant improvement of pain $(\mathrm{p}<0.05)$ compared to McKenzie approach. ${ }^{9}$ The effects of lumbar stabilization exercises were compared with lumbar dynamic strengthening exercises on pain severity of patients with chronic low back pain and their functional disability in RCT and it was reported that the patients in lumbar stabilization exercises group showed improved strength and functional outcomes compared to other therapies applied for the treatment of chronic low back pain. ${ }^{10}$

A study conducted Hossainifar et al. concluded that stabilization exercises are way more effective than McKenzie in improving functional abilities and pain scores in non-specific chronic low back pain. This was an RCT where the effects of stabilization and McKenzie exercises were compared on TrA and multifidi muscles thickness, pain and disability. ${ }^{11}$ Similar findings were reported in a systematic review which included 18 RCTs where the patients receiving stabilization exercises showed superior results in patients with chronic low back pain. This study supports the current study results that stabilization exercises improve pain and function in patients with chronic low back pain. ${ }^{12}$

In a systematic review by Keating et al. on trunkstrengthening exercises for chronic low back pain it was reported that that trunk strengthening exercises showed good outcomes including inspiration increment treatment impacts. Trunk reinforcing, contrasted and aerobics exercise or McKenzie works out, demonstrated relatively small advantage of strengthening. ${ }^{13}$

An RCT with a 1-year follow-up supported our study findings that the orthopaedic manual treatment and McKenzie techniques appeared to be more successful treatment protocols. ${ }^{14}$ The effectiveness of McKenzie method in addition to first-line care for acute low back pain was reported in an RCT carried out by Machado et al. The study suggested that first-line care of intense low back pain did not show reasonable effects for reducing pain when treated with McKenzie protocols. It is obvious from the literature that McKenzie protocols appears to be relatively inferior than the stabilization techniques for the treatment of chronic low back pain. ${ }^{15}$

A systematic review on randomized controlled trials by Rackwitz et al. supported the results of current study that segmental stabilization exercises are more effective than the other therapies applied for the management of chronic low back pain. ${ }^{16}$

CONCLUSION

The study results conclude that both treatment groups showed improvement by reducing pain and improving functional status outcomes, however, lumbar stabilization exercises are more effective in the management of chronic low back pain compared to McKenzie approach.

REFERENCES

1. Hartvigsen J, Hancock MJ, Kongsted A, et al. What low back pain is and why we need to pay attention. The Lancet. 2018;391(10137):2356-2367.

2. Synnott A, O'Keeffe M, Bunzli S, Dankaerts W, O'Sullivan P, O'Sullivan K. Physiotherapists may stigmatise or feel unprepared to treat people with low back pain and psychosocial factors that influence recovery: a systematic review. Journal of physiotherapy. 2015;61(2):68-76.

3. Richardson C, Jull G, Hodges P, Hides J. Therapeutic Exercise for Spinal Segmental Stabilization in Low Back Pain: Scientific Basis and Clinical Approach. 1999;

4. Standaert CJ, Weinstein SM, Rumpeltes J. Evidence-informed management of chronic low back pain with lumbar stabilization exercises. The spine journal. 2008;8(1):114-120.

5. França FR, Burke TN, Hanada ES, Marques AP. Segmental stabilization and muscular strengthening in chronic low back pain: a comparative study. Clinics. 2010;65(10):1013-1017.

6. Shaughnessy M, Caulfield B. A pilot study to investigate the effect of lumbar stabilisation exercise training on functional ability and quality of life in patients with chronic low back pain. International journal of rehabilitation research. 2004;27(4):297-301.

7. Kahl C, Cleland JA. Visual analogue scale, numeric pain rating scale and the McGill Pain Questionnaire: an overview of psychometric properties. Physical therapy reviews. 2005;10(2):123-128.

8. Dillard J, Trafimow J, Andersson G, Cronin K. Motion of the lumbar spine. Reliability of two measurement techniques. Spine. 1991;16(3):321-324.

9. Miller ER, Schenk RJ, Karnes JL, Rousselle JG. A Comparison of the McKenzie Approach to a Specific Spine Stabilization Program for Chronic Low Back Pain. Journal of Manual \& Manipulative Therapy. 2005/04/01 2005;13(2):103-112. doi:10.1179/106698105790824996 10. Moon HJ, Choi KH, Kim DH, et al. Effect of lumbar stabilization and dynamic lumbar strengthening exercises in patients with chronic low back pain. Ann Rehabil Med. doi:10.5535/arm.2013.37.1.110

11. Hosseinifar M, Akbari M, Behtash H, Amiri M, Sarrafzadeh J. The Effects of Stabilization and Mckenzie Exercises on Transverse Abdominis and Multifidus Muscle Thickness, Pain, and Disability: A Randomized Controlled Trial in NonSpecific Chronic Low Back Pain. Journal of Physical Therapy Science. 2013;25(12):15411545. doi:10.1589/jpts.25.1541

12. May S, Johnson R. Stabilisation exercises for low back pain: a systematic review. Physiotherapy. 2008/09/01/ 2008;94(3):179-189. doi:https://doi.org/10.1016/j.physio.2007.08.010

13. Slade SC, Keating JL. Trunk-Strengthening Exercises for Chronic Low Back Pain: A Systematic Review. Journal of Manipulative and Physiological Therapeutics. 2006/02/01/ 2006;29(2):163-173. doi:https://doi.org/10.1016/j.jmpt.2005.12.011

14. Paatelma M, Kilpikoski S, Simonen R, Heinonen A, Alen M, Videman T. Orthopaedic manual therapy, McKenzie method or advice only for low back pain in working adults: a randomized controlled trial with one year follow-up. Journal of rehabilitation medicine. 2008;40(10):858-863.

15. Machado LAC, Maher CG, Herbert RD, Clare $\mathrm{H}$, McAuley JH. The effectiveness of the McKenzie method in addition to first-line care for acute low back 
pain: a randomized controlled trial. BMC Medicine. 2010/01/26 2010;8(1):10. doi:10.1186/1741-7015-8-10 16. Rackwitz B, de Bie R, Limm H, von Garnier K, Ewert T, Stucki G. Segmental stabilizing exercises and low back pain. What is the evidence? A systematic review of randomized controlled trials. Clinical rehabilitation. 2006;20(7):553-567. 


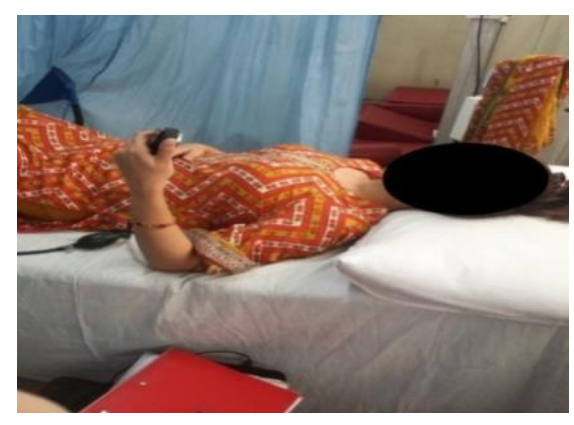

Figure-1 TrA Activation
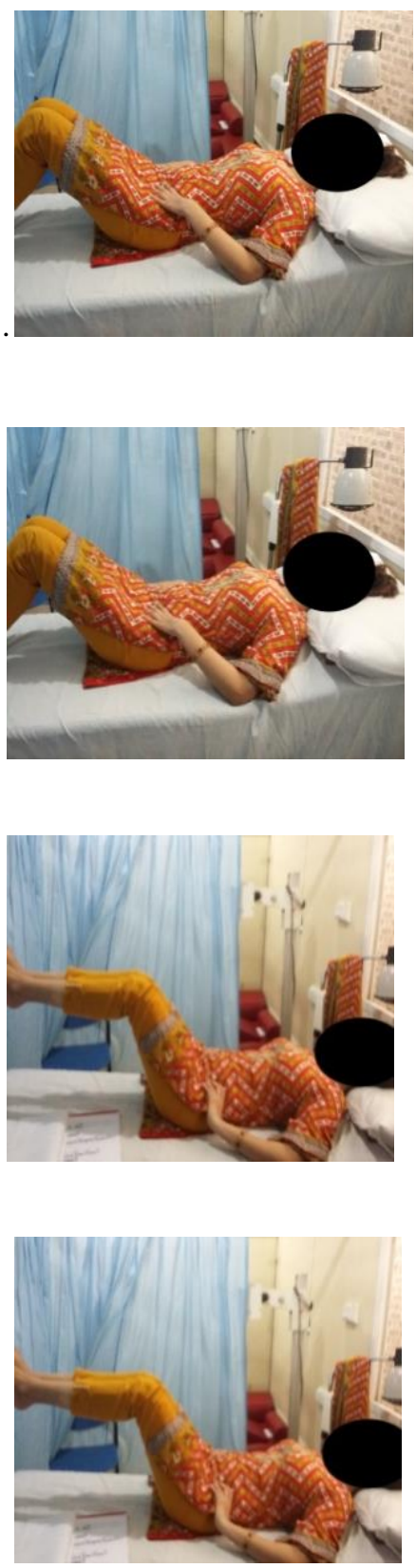

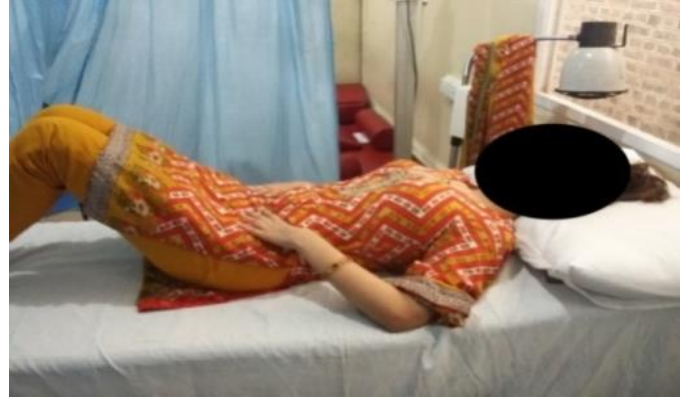

Figure-2 Level 1 TrA

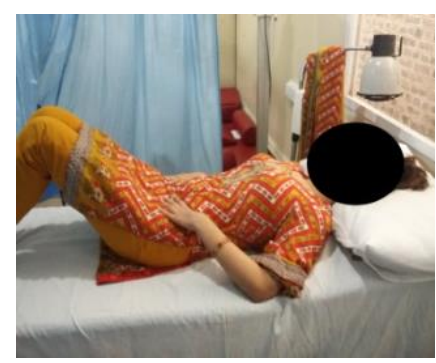

Figure 3 Level 2 TrA
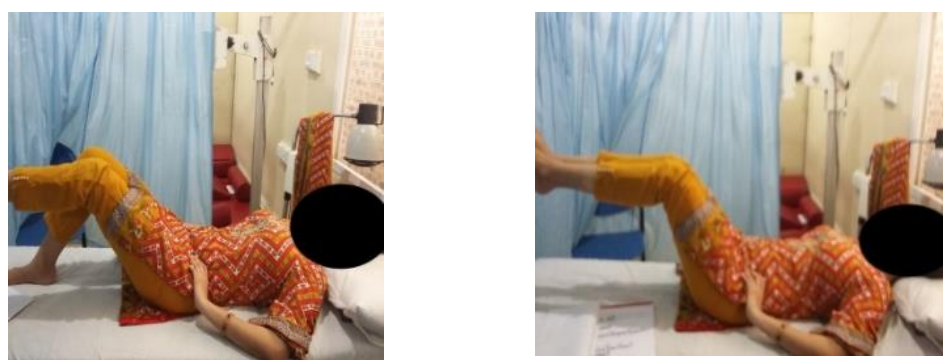

Figure 4: Figure showing level 3

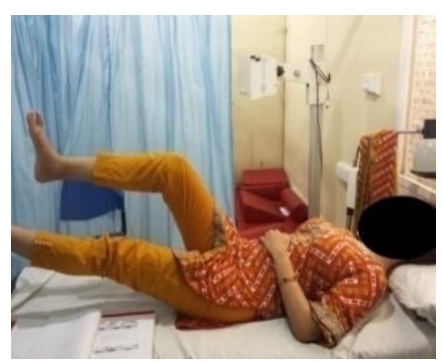

Figure 5: Level 4

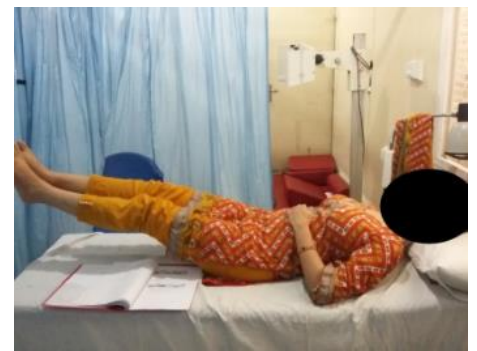

Figure 6: Level 5
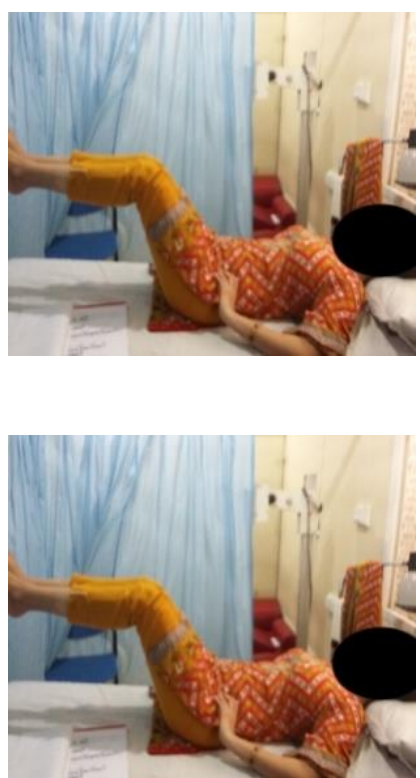


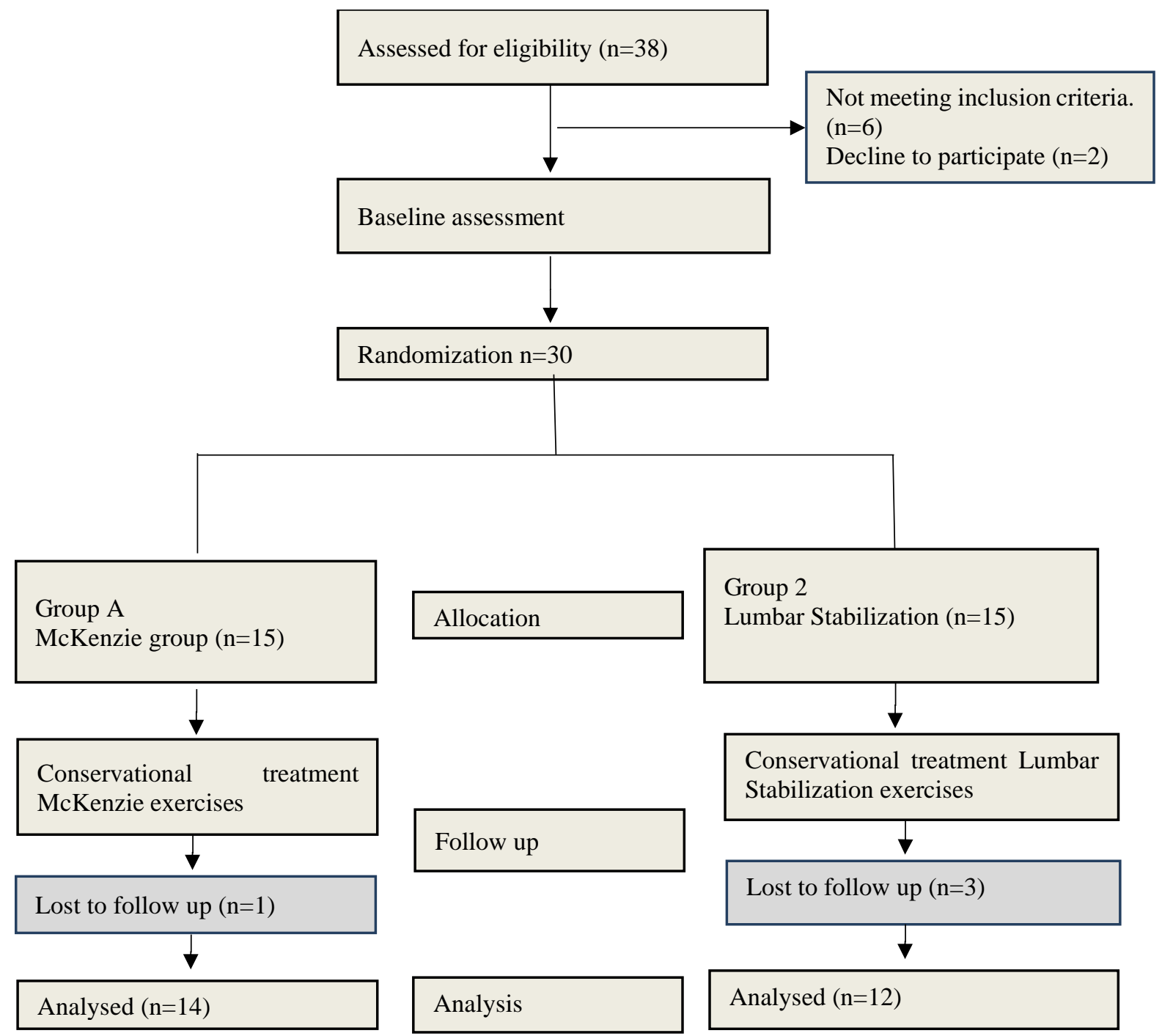

Figure 7: Consort flowchart showing patients recruitment

Table 1: Demographics of the included patients

\begin{tabular}{|lll|}
\hline Variable & & Frequency/ \%age \\
Age (Years) & McKenzie & $52.42 \pm 12.29$ \\
\multirow{2}{*}{ Nature of Pain } & LS group & $49 \pm 12.38$ \\
& Localized & $19(70.4 \%)$ \\
Characteristics of Pain & Radiating & $7(29.6 \%)$ \\
& Continuous & $7(29.6 \%)$ \\
& Intermittent & $13(48.2 \%)$ \\
Any Ongoing Treatment & Exercise & $1(3.7 \%)$ \\
& Rest & $10(37 \%)$ \\
& Medicine & $12(44 \%)$ \\
\hline
\end{tabular}

$\mathrm{LS}=$ Lumber Stabilization

Table 2: Baseline assessment results of both groups of the selected outcomes

\begin{tabular}{|lllll|}
\hline Variables & Groups & Mean rank & Median (IQ) & P value \\
NPRS & McKenzie & 14.79 & $6(1)$ & 0.390 \\
ROM flexion & LS & 12.39 & & \\
& McKenzie & 14.42 & $40(2)$ & 0.568 \\
ROM extension & LS & 12.71 & & 0.896 \\
& McKenzie & 13.29 & $15(3)$ & \\
\hline
\end{tabular}




\begin{tabular}{|lllll|}
\hline \multirow{2}{*}{ ROM Rt. Side bending } & McKenzie & 14.21 & $15(5.25)$ & 0.655 \\
& LS & 12.89 & & \\
ROM Left side bending & McKenzie & 12.96 & $15(5.5)$ & 0.734 \\
& LS & 13.96 & $49(20.5)$ & 0.696 \\
\hline
\end{tabular}

ROM= Range of Motion

Table 3: Within Group Analysis (McKenzie Group)

\begin{tabular}{|c|c|c|c|c|}
\hline Variables & Groups & Median (IQ) & Mean Rank & $P$ value \\
\hline NPRS & $\begin{array}{l}\text { Pre-intervention } \\
\text { Post-intervention }\end{array}$ & $\begin{array}{l}6.5(1) \\
3(2)\end{array}$ & 7.50 & 0.000 \\
\hline ROM flexion & $\begin{array}{l}\text { Pre-intervention } \\
\text { Post-intervention }\end{array}$ & $\begin{array}{l}36(15) \\
56(5)\end{array}$ & 7.50 & 0.000 \\
\hline ROM extension & $\begin{array}{l}\text { Pre-intervention } \\
\text { Post-intervention }\end{array}$ & $\begin{array}{l}17(6.75) \\
25(3)\end{array}$ & 7.50 & 0.000 \\
\hline ROM rt. bending & $\begin{array}{l}\text { Pre-intervention } \\
\text { Post-intervention }\end{array}$ & $\begin{array}{l}15(4.5) \\
20(5.5)\end{array}$ & 6.50 & 0.000 \\
\hline ROM left bending & $\begin{array}{l}\text { Pre-intervention } \\
\text { Post-intervention }\end{array}$ & $\begin{array}{l}15.5(6.25) \\
21(3.5)\end{array}$ & 7.00 & 0.000 \\
\hline ODI & $\begin{array}{l}\text { Pre-intervention } \\
\text { Post-intervention }\end{array}$ & $\begin{array}{l}49(20.25) \\
28(9.25)\end{array}$ & 7.50 & 0.000 \\
\hline
\end{tabular}

Table 4: Within Group Analysis (LS Group)

\begin{tabular}{|c|c|c|c|c|}
\hline Variables & Groups & Median (IQ) & Mean Rank & $\mathrm{P}$ value \\
\hline \multirow{2}{*}{ NPRRS } & Pre-intervention & $6(1)$ & \multirow{2}{*}{6.50} & \multirow{2}{*}{0.000} \\
\hline & Post-intervention & $3(1)$ & & \\
\hline \multirow{2}{*}{ ROM flexion } & Pre-intervention & $42(13)$ & \multirow{2}{*}{6.50} & \multirow{2}{*}{0.000} \\
\hline & Post-intervention & $53(7.25)$ & & \\
\hline \multirow{2}{*}{ ROM extension } & Pre-intervention & $14.5(6)$ & \multirow{2}{*}{6.50} & \multirow{2}{*}{0.000} \\
\hline & Post-intervention & $20(3)$ & & \\
\hline \multirow{2}{*}{ ROM rt. Side bending } & Pre-intervention & $15(7.5)$ & \multirow{2}{*}{5.50} & \multirow{2}{*}{0.000} \\
\hline & Post-intervention & $19(6.5)$ & & \\
\hline \multirow{2}{*}{ ROM left side bending } & Pre-intervention & $15(6.5)$ & \multirow{2}{*}{5.50} & \multirow{2}{*}{0.000} \\
\hline & Post-intervention & $19(5.5)$ & & \\
\hline \multirow{2}{*}{ ODI } & Pre-intervention & $49(20.75)$ & \multirow{2}{*}{6.50} & \multirow{2}{*}{0.000} \\
\hline & Post-intervention & $25(8.75)$ & & \\
\hline
\end{tabular}

Table 5: Post Intervention Analysis Between Groups

\begin{tabular}{|c|c|c|c|c|}
\hline Variables & Groups & mean rank & $\overline{\text { Median (IQ) }}$ & $P$ value \\
\hline \multirow{2}{*}{ NPRS } & McKenzie group & 17.33 & \multirow{2}{*}{$3(1.5)$} & \multirow{2}{*}{0.001} \\
\hline & LS Group & 10.21 & & \\
\hline \multirow{2}{*}{ ROM flexion } & McKenzie Group & 10.46 & \multirow{2}{*}{$55(6.2)$} & \multirow{2}{*}{0} \\
\hline & LS Group & 16.11 & & \\
\hline \multirow{2}{*}{ ROM extension } & McKenzie Group & 9.04 & \multirow{2}{*}{$23(5)$} & \multirow{2}{*}{0.004} \\
\hline & LS Group & 17.32 & & \\
\hline \multirow{2}{*}{ ROM rt. Side bending } & McKenzie Group & 11.75 & \multirow{2}{*}{$19.5(3.5)$} & \multirow{2}{*}{0.275} \\
\hline & LS Group & 15 & & \\
\hline \multirow{2}{*}{ ROM left side bending } & McKenzie Group & 11.21 & \multirow{2}{*}{$20(4.5)$} & \multirow{2}{*}{0.15} \\
\hline & LS Group & 15.46 & & \\
\hline \multirow{2}{*}{ ODI } & McKenzie Group & 14.42 & \multirow{2}{*}{$26(11)$} & \multirow{2}{*}{0.07} \\
\hline & LS Group & 12.71 & & \\
\hline
\end{tabular}

